\title{
A cardiac signature of emotionality
}

\author{
Stefan Koelsch, ${ }^{1,2}$ Andrew Remppis, ${ }^{3}$ Daniela Sammler, ${ }^{1}$ Sebastian Jentschke, ${ }^{1}$ Daniel Mietchen, ${ }^{1}$ Thomas Fritz, ${ }^{1}$ \\ Hendrik Bonnemeier ${ }^{4}$ and Walter A. Siebel ${ }^{5}$ \\ ${ }^{1}$ Max Planck Institute for Human Cognitive and Brain Sciences, Leipzig, Germany \\ ${ }^{2}$ University of Sussex, Department of Psychology, Brighton, UK \\ ${ }^{3}$ Medizinische Klinik III, University of Heidelberg, Germany \\ ${ }^{4}$ Medizinische Klinik II, University of Lübeck, Germany \\ ${ }^{5}$ Conflict Research Center, Wiesbaden, Germany
}

Keywords: amygdala, emotion, fMRI, hippocampus, HRV, hyperathymia maxima, personality

\begin{abstract}
Human personality has brain correlates that exert manifold influences on biological processes. This study investigates relations between emotional personality and heart activity. Our data demonstrate that emotional personality is related to a specific cardiac amplitude signature in the resting electrocardiogram (ECG). Two experiments using functional magnetic resonance imaging show that this signature correlates with brain activity in the amygdala and the hippocampus during the processing of musical stimuli with emotional valence. Additionally, this cardiac signature correlates with subjective indices of emotionality (as measured by the Revised Toronto Alexithymia Scale), and with both time and frequency domain measures of the heart rate variability. The results demonstrate intricate connections between emotional personality and the heart by showing that ECG amplitude patterns provide considerably more information about an individual's emotionality than previously believed. The finding of a cardiac signature of emotional personality opens new perspectives for the investigation of relations between emotional dysbalance and cardiovascular disease.
\end{abstract}

\section{Introduction}

Personality is not only a psychological but also a biological phenomenon: An individual's personality is related to numerous neurobiological processes, including regulation of neurotransmitter systems (Hollander \& Evers, 2001; Carver \& Miller, 2006; Netter, 2006), hormones (Zuckerman et al., 1980; Rubinow \& Schmidt, 1996; Biondi \& Picardi, 1999; Griffiths et al., 2000), as well as of autonomic (Eysenck, 1990; Lorber, 2004) and immune system activity (Adler \& Matthews, 1994; Segerstrom, 2000; Kiecolt-Glaser et al., 2002a,b; Ader, 2006). Personality is also related to the activity of peripheral organs, especially via the hormonal and the autonomic nervous systems (Verrier \& Mittelman, 2000; Armour \& Ardell, 2004). The organ that has been most extensively investigated in this regard is the heart. It is well documented that emotional processes affect a variety of parameters of heart activity, such as heart rate (HR), heart rate variability (HRV) and contractility (Cacioppo et al., 2000; Verrier \& Mittelman, 2000; Armour \& Ardell, 2004). A substantial body of evidence indicates that affective disorders such as anxiety and depressive disorders are associated with reduced HRV, as well as reduced QT variability (Friedman \& Thayer, 1998; Yeragani et al., 1993, 2000; Carney et al., 2001), and that chronic negative emotions such as fear, hostility, depression and anger increase the risk for coronary heart disease and heart attack (Lesperance et al., 1996; Rozanski et al., 1999; Kubzansky \& Kawachi, 2000; MacMahon \&

Correspondence: Dr S. Koelsch, as above. ${ }^{1}$

E-mail: koelsch@cbs.mpg.de

Received 18 December 2006, revised 11 September 2007, accepted 17 September 2007
Lip, 2002; Singh et al., 2002; Joynt et al., 2004; Smith et al., 2004; Rozanski \& Kubzansky, 2005; Stanton et al., 2007).

The present study aims at examining relations between amplitudes of the electrocardiogram (ECG) and emotional personality. The type of emotional personality we investigated is denoted here as tender positive emotionality. It refers to the capability of producing tender positive feelings that can be described, for example as soft, loving, warm and joyful (as opposed to, e.g. aggressive, impulsive, hostile, anxious, manic or depressive affect). Tender positive emotionality (or its absence) may become apparent in facial expression, prosody, gestural expression and use of tender emotional language (for evaluation of emotional expression in these domains, see Andreasen, 1982; Russell \& Fernández-Dols, 1997; Lundh \& Simonsson-Sarnecki, 2001; Pell, 2001). It is reminiscent of the facets positive emotion, warmth and tender-mindedness of the Revised Neuroticism-Extraversion-Openness Personality Inventory (NEO PI-R; Costa \& McCrae, 1992), where positive emotion is described as tendency to experience positive emotions, warmth as interest in and friendliness towards others, and tender-mindedness as attitude of sympathy for others (ibid.). However, in contrast to the NEO PI-R constructs warmth and tender-mindedness, the concept of tender positive emotionality puts more emphasis on emotionality than on attitudes or behaviour, and considers that tender emotionality can also be directed towards oneself.

Quantitative measures for the assessment of tender positive emotionality have, to our knowledge, not been reported so far, although several diseases and disorders are related to a lack of positive emotions, e.g. depression, chronic fear, Alexithymia (Taylor, 1984; Taylor \& Bagby, 2004), and hyperathymia maxima (Siebel, 

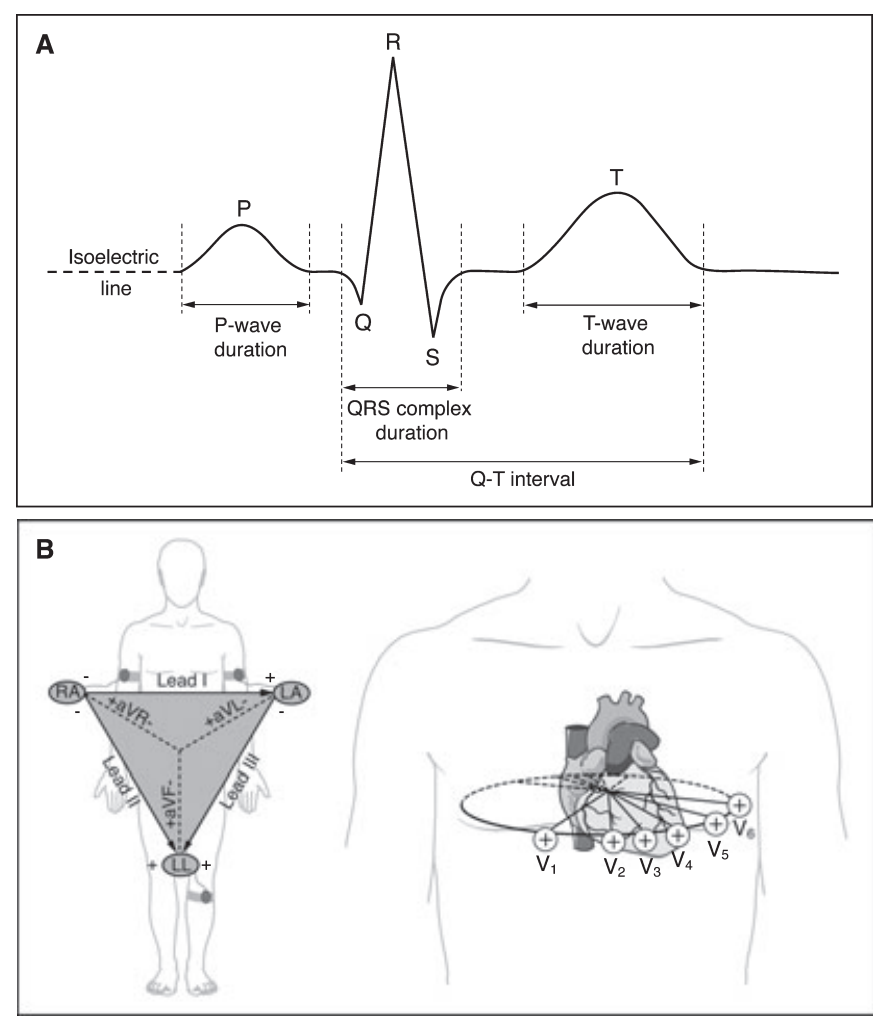

FIG. 1. Basic ECG waves of a normal heartbeat (A), the P-wave reflects atrial depolarization, the QRS complex depolarization of the ventricles, and the T-wave ventricular repolarization. Standard ECG leads are shown in (B). The six extremity leads (I, II, III, aVL, aVR, aVF) record voltage differences by means of electrodes placed on the limbs (left panel of B). The triangle shows the spatial relationships of the extremity leads, which record electrical voltages onto the frontal plane of the body. The six chest leads (V1-V6) record voltage differences by means of electrodes placed on the chest wall (right panel of B). The oval indicates spatial relationships of the six chest leads, which record electrical voltages transmitted onto the horizontal plane.

1994; Siebel \& Winkler, 1996). We investigated correlations between tender positive emotionality and interindividual differences in the amplitude patterns of ECG waves (see Fig. 1A for illustration of ECG waves), which have been proposed to indicate emotional personality (Siebel \& Winkler, in press). In Experiment 1, we demonstrate relations between a cardiac amplitude signature and emotional personality. In Experiment 2, we psychologically validate this finding. Experiment 3 shows that the cardiac signature identified in Experiment 1 predicts the amount of activity changes in an individual's limbic brain structures (amygdala and hippocampus) during the processing of stimuli with emotional valence. Experiment 4 shows that this cardiac signature is also related to the autonomic balance of individuals as indicated by HRV measures.

\section{Experiment 1: a cardiac signature of emotionality}

Experiment 1 was set out to identify relations between emotionality and ECG amplitude ratios. Therefore, we acquired from each individual a resting ECG, a narrative interview designed to obtain a score of tender emotionality, and Alexithymia scores as measured with the Revised Toronto Alexithymia Scale (see Materials and methods for additional measures). Discriminant function analysis was then performed to identify ECG amplitude patterns that correlate with emotional personality.

\section{Materials and methods, Experiment 1}

Subjects

Eighty healthy individuals without psychiatric disorders (age range 21-39 years, mean 25.3 years, 40 females) participated in the experiment. As for all of the following experiments, informed consent was obtained from all participants. The study was conducted in accordance with the Declaration of Helsinki.

\section{Procedure}

After the ECG measurements (see below), body height and body weight were obtained, then participants filled out the German version of the Revised Toronto Alexithymia Questionnaire (TAS-26; Taylor et al., 1992; Kupfer et al., 2000). This questionnaire consists of three subscales: (1) the ability to identify one's feelings; (2) difficulties in describing emotions; and (3) external thinking. We were interested in the scores of the first TAS-scale (TAS-1), because this subscale has been reported to capture emotional impairment better than the two other subscales (Haviland et al., 1988).

Subsequently, each participant underwent a narrative interview, in which participants were asked to report three positive events. Based on these interviews, the emotionality of subjects was rated on six visual analogue scales by one of the authors (S.K.; in Experiment 2, emotionality assessments will be performed by multiple raters): (1) reports of own tender emotions; (2) use of tender emotional language; (3) facial and eye expression of tender emotion; (4) prosodic expression of tender emotion; (5) gestural expression of tender emotion; and (6) global rating of tender emotionality. Scales 1 and 2 were used following the Alexithymia construct (Taylor, 1984; Lundh \& Simonsson-Sarnecki, 2001), and scales 3-6 were adopted from the Affective Flattening subscale of the Scale for the Assessment of Negative Symptoms (SANS; Andreasen, 1982; the SANS itself was not used because it is designed to assess clinical symptoms of psychotic patients). Each visual-analogue scale consisted of 50 adjacent circles, with the outermost left circle representing very low tender emotionality, and the outermost right circle very high tender emotionality. For each participant, ratings on the six scales were averaged, resulting in an emotionality score for each subject (scores with high values corresponded to ratings of high tender emotionality, and vice versa).

\section{ECG measurements}

A 12-lead resting ECG (2 min duration) was obtained from each participant placed in a supine position under standard conditions with a sampling rate of $1000 \mathrm{~Hz}$ (see Fig. 1B for illustration of ECG leads). For each lead of each participant, 100 P-, T- and R-waves were marked at their maximum amplitude in the raw ECG, and averaged (separately for each lead) to increase the signal-to-noise ratio. Then, amplitudes of ECG waves were measured in the following way (for similar methods, see Pan et al., 1992; McLaughlin et al., 1995; Soria-Olivas et al., 1998; Martinez \& Olmos, 2005): the R-peak amplitudes were measured with respect to the baseline of the averaged ECG cycle (this baseline was calculated by computing the mean amplitude value of the time interval from 350 to $300 \mathrm{~ms}$ preceding the R-peak). $\mathrm{P}$-wave amplitudes were measured as the absolute difference between P-wave peak amplitude and the baseline of the ECG cycle (this baseline was calculated by computing the mean amplitude value of the time interval from 180 to $130 \mathrm{~ms}$ preceding the peak of the $\mathrm{P}$-wave). Measurement of T-wave amplitudes aimed at finding the difference between the T-wave peak and the first plateau-like wave shape preceding the T-wave peak (for similar methods, see Pan et al., 1992; McLaughlin et al., 1995; Martinez \& Olmos, 2005). Therefore, the 
first derivative of the waveform was calculated (plateau-like wave shapes result in small values of the first derivative), and the standard deviation of the first derivative within the 100-ms time-window preceding the T-wave peak $\left(\sigma_{100}\right)$ was computed. The value of the first sampling point of the ECG wave (preceding the T-wave peak) for which the value of the first derivative was smaller than $1 / 20$ of $\sigma_{100}$ was then subtracted from the value of the T-wave peak (if this point was located within the QRS-complex, the factor of $1 / 20$ was enlarged until this point was located beyond the QRS complex). RS-complexes were measured as the absolute difference between R- and S-waves (if there was more than one R- or S-wave, only the largest R- and S-peaks were taken into account). Amplitude values of ECG waves were measured electronically using Kardionoon 1.0 (http://www.kardionoon.net) and Matlab7.0 (MathWorks, Natick, MA, USA). (The Matlab-Script for the measurements of ECG amplitudes is available on request.) All measured (absolute) amplitude values were entered into the discriminant function analysis. To compensate for differences in the positioning of chest leads between subjects, maxima of absolute values of P-, T- and R-waves, as well as of RS-complexes, were calculated for all chest leads (and added as variables to the discriminant function analysis).

\section{Results, Experiment 1}

\section{Emotionality and ECG amplitude ratios}

Emotionality scores as obtained from the narrative interviews had a range from 7 to $45(M=30, S D=12)$. These scores were ranked, and for the lower and upper quartile of participants amplitudes of ECG waves from all leads were subjected to a stepwise discriminant function analysis (probability for entry: $P=0.05$; for removal: $P=0.10$; body height, body weight and body mass index were also entered as variables). The analysis identified four ECG waves as discriminating variables: the RS complex of the aVL lead, the T wave of the aVL lead, the R wave of lead III, and the maximal RS complex of the chest leads (Fig. 2), 100\% of cross-validated grouped cases were correctly classified, and group centroids significantly differed from each other (group centroids were -2.6 for low, and 2.6 for high emotionality; $\chi_{4}^{2}=74.6 ; P<0.0001$; Wilks' $\Lambda=0.13$ ). This finding indicates that a combination of ECG amplitude values specifically varies as a function of an individual's emotionality.

\section{Computation of $E_{\mathrm{\kappa}}$ values}

Discriminant function analysis, however, is based on a weighted linear combination of ECG amplitudes, which are dependent on factors such as body size and electric resistance of the body tissues between heart and ECG leads. These factors substantially differ between individuals, but are most likely unrelated to differences in emotionality (assuming that an individual's emotionality is not related, e.g. to body height). To cancel down such factors, we computed ratios of ECG amplitudes by placing ECG amplitudes with positive discriminant function coefficients (the T wave of the aVL lead and the R wave of lead III) in the nominator, and those with negative ones (the RS complex of the aVL lead and the maximal RS complex of the chest leads) in the denominator (Fig. 2C). The result of this equation is an ECG-based index $E_{\kappa}$, with the advantage of a minimized dependence on interindividual differences in tissue conductivity or strength of the electric heart vector (given similar vector orientations). Confirming this assumption, $E_{\kappa}$ values of participants did not correlate with body mass index, body weight or body height $(P>0.49$ in each test).

In the group quartile with low emotionality, $E_{\mathrm{\kappa}}$ values resulting from this computation had a range of $0.04-0.32$ (mean $=0.14$,
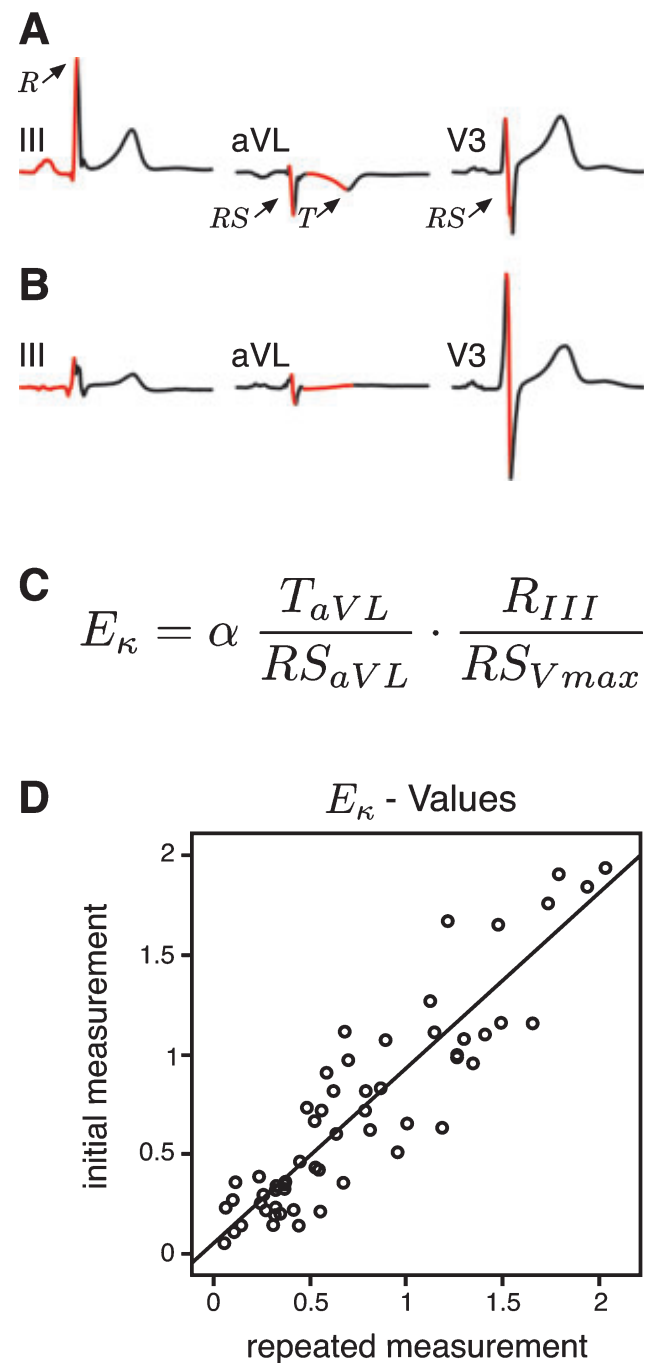

FIG. 2. Cardiac signatures of emotionality: Averaged ECG cycles measured from leads III, aVL and V3, plotted separately for a subject with high (A) and low emotionality (B), ECG amplitudes identified by the discriminant function analysis as discriminating variables are indicated in red: the R-wave of III ( $\mathrm{R}_{\mathrm{III}}$, measured from the baseline preceding the P-wave), the T-wave of aVL $\left(\mathrm{T}_{\mathrm{aVL}}\right)$, the RS-complex of aVL $\left(\mathrm{RS}_{\mathrm{aVL}}\right)$, and the RS-complex of the chest lead with the maximal RS amplitude $\left(\mathrm{RS}_{\mathrm{V} \max }\right)$. For each subject, the cardiac emotionality index $E_{\mathrm{K}}$ is calculated using the formula shown in (C) (for a better readability of $E_{\kappa}$ values, values are scaled by a factor $\alpha=10$ ). The application of this formula to the ECG amplitudes extracted from the recordings shown in (A) and (B) results in an $E_{\kappa}$ value of 2.34 for subject A (high emotionality), and of 0.16 for subject $B$ (low emotionality). (D) The test/retest reliability of the ECG amplitude signature: Each circle represents $E_{\kappa}$ values of one subject obtained in an initial measurement [vertical axis] and a repeated measurement [horizontal axis]. Pearson's test/retest correlation was $r=0.90(P<0.0001)$.

$\mathrm{SD}=0.08), E_{\kappa}$ values in the group quartile with high emotionality had a range of $0.81-2.06$ (mean $=1.32, \mathrm{SD}=0.37)$. That is, notably all $E_{\mathrm{\kappa}}$ values in the quartile with low emotionality were distinctly lower than in the quartile with high emotionality. A $t$-test comparing the $E_{\kappa}$ values between group quartiles indicated a significant difference $\left(t_{38}=14.1, P<0.0001\right)$.

\section{$E_{\kappa}$ values and questionnaire scores}

In addition to the interviews and ECGs, we also obtained an index of emotionality with the TAS-1 scale (see Materials and methods, Experiment 1). The 80 subjects were divided into two groups (high, low $E_{\kappa}$ values) based on a median split of $E_{\kappa}$ values (median was 
0.42). In the group of subjects with lower $E_{\kappa}$ values, TAS-1 scores of four subjects (out of 40 ) were one standard deviation above the mean of the norm population (i.e. $t$-values of TAS-1 scores above 60 ); in the group of subjects with higher $E_{\kappa}$ values, two (out of 40) subjects had TAS-1 scores outside this range (the German translation of the TAS-26 also provides $t$-values, unlike the original TAS-26; with regards to the total TAS scores, four individuals of the group with lower, and three individuals of the group with higher $E_{\kappa}$ values lay within the range of Alexithymia). However, when taking TAS-1 scores into account that were one standard deviation above or below the mean of the norm population (i.e. $t$-values of TAS- 1 scores either above 60 or below 40), TAS-1 scores of 13 subjects (out of 40 ) were outside this range in the group of subjects with lower $E_{\mathrm{\kappa}}$ values, whereas only three (out of 40) were outside this range in the group of subjects with higher $E_{\mathrm{\kappa}}$ values, the difference between groups being significant (Pearson $\chi_{1}^{2}=7.81$, $P<0.005)$. That is, the prevalence of TAS- 1 scores outside the norm range was higher in individuals with low than in those with high $E_{\kappa}$ values.

\section{Reliability of $E_{\mathrm{\kappa}}$ values}

To evaluate the reliability of $E_{\kappa}$ values, retest sessions were performed in which ECGs were measured from 55 of the 80 subjects participating in Experiment 1 (28 of them were females, age range was 2036 years, mean age 24.9 years). Test and retest sessions were separated by at least 6 weeks. A Pearson's test/retest correlation between the $E_{\mathrm{\kappa}}$ values of test and retest sessions revealed a remarkably high reliability of these values $(r=0.90, P<0.0001$; Fig. 2D). This renders it impossible that factors such as normal variations in electrode placement or normal circadian fluctuations in autonomic tone considerably influence the $E_{\kappa}$ values.

\section{Discussion, Experiment 1}

The $E_{\mathrm{\kappa}}$ values, which were derived by computing the coefficients provided by the discriminant analysis, clearly differed between the group quartile with high and the group quartile with low emotionality. This indicates that a combination of ECG amplitude values specifically varies as a function of an individual's emotional personality. Interestingly, the ECG pattern of individuals with low $E_{\mathrm{\kappa}}$ values clearly resembles the ECG pattern described for individuals with hyperathymia maxima (a putative disorder associated with persistent lack of tender positive emotionality; Siebel \& Winkler, 1996; Siebel \& Winkler, in press). Furthermore, the finding that interindividual differences in emotionality are related to differences in ECG amplitude patterns is supported by the observation that more than $90 \%$ of the individuals with higher $E_{\mathrm{\kappa}}$ values had TAS-1 T-scores within the norm range, whereas about one-third of the individuals with lower $E_{\kappa}$ values had TAS-1 T-scores outside the norm range. This also corroborates the assumption that lower $E_{\kappa}$ values correlate with aberrant emotionality. The finding that the ECG amplitude patterns correlate with emotional personality opens the interesting possibility to use ECG data as an index of an individual's emotionality. Compared with subjective measures (such as the TAS), the ECG measure has the advantage of being independent of subjective bias inherent to subjects and investigators (but see also General discussion).

\section{Experiment 2: validity of the cardiac signature}

Experiment 2 aimed at testing whether the cardiac signature identified in Experiment 1 also correlates with differences in emotional personality when subjects' emotionality is rated by multiple raters.
Here, an independent group of 36 participants estimated each other's emotionality, so that average group ratings of the emotionality for each individual were obtained, with the rationale that errors inherent in individual ratings may tend to cancel out across individuals (Antaki et al., 1988). We hypothesized that emotionality scores would positively correlate with the $E_{\mathrm{\kappa}}$ values of subjects.

\section{Materials and methods, Experiment 2}

\section{Subjects}

Thirty-six healthy individuals without psychiatric disorders (age range 20-31 years, mean 26 years, 18 females) participated in the experiment. None of the individuals had participated in Experiment 1. Participants were students of a psychology seminar on physiological correlates of emotion and emotional personality. In the course of the seminar, the students were made acquainted with each other, and during two seminar sessions prior to the rating, the students were familiarized with the concept of tender positive emotionality.

Rest-ECGs were obtained as in Experiment 1. One ECG was excluded from further analysis due to artefacts. Each participant had a questionnaire with one visual analogue scale for each of the remaining 35 participants. Subjects were asked to rate the global tender emotionality of the other participants on these scales. Visual analogue scales consisted of 50 adjacent circles, with the outermost left circle representing very low tender emotionality, and the outermost right circle very high tender emotionality. Individual ratings were then averaged, resulting in a score of emotionality for each participant. None of the students was informed about the study prior to the rating procedure (to ensure as high an independence of individual ratings as possible), and none of the participants was familiar with any $E_{\kappa}$ value prior to the rating procedure.

\section{Results and discussion, Experiment 2}

Emotionality scores ranged from 19.1 to $35.2(\mathrm{M}=27.6, \mathrm{SD}=3.8)$, $E_{\mathrm{\kappa}}$ values from 0.04 to $2.4(\mathrm{M}=0.72, \mathrm{SD}=0.55)$. A non-parametric rank correlation of the emotionality scores and the $E_{\mathrm{\kappa}}$ values indicated a significant positive correlation (Spearman's $\rho=0.46, P<0.005$ ). That is, the $E_{\mathrm{\kappa}}$ values significantly predicted the emotionality scores of subjects (as obtained by the averaged emotionality ratings). In other words, the cardiac signature of an individual predicts how the emotionality of this individual would be rated by a number of independent subjects. This finding validates the relation between emotionality and the ECG amplitude signature observed in Experiment 1.

\section{Experiment 3a: relations between the cardiac signature and emotional brain activity}

To further validate the relation between this cardiac signature and emotional personality, we carried out two experiments using functional magnetic resonance imaging (fMRI) in which $E_{\kappa}$ values could be related to activity in brain structures known to be involved in the processing of emotion. The fMRI experiments were designed to elicit emotional brain activity in response to pleasant and unpleasant music. Resting ECGs of participants were measured at least 1 day prior to the functional imaging experiments (that is, ECGs were not measured inside the MR scanner, and no stimulation was applied during ECG measurements), $E_{\kappa}$ values were then computed for each participant according to the formula described in Fig. 2C. 
The first fMRI experiment (Experiment 3a) used an experimental protocol identical to a previous study (Koelsch et al., 2006). Changes in blood oxygen level-dependent (BOLD) signals were measured from 17 participants during the presentation of musical stimuli that had either a positive (pleasant) or a negative (unpleasant) emotional valence. The above-mentioned fMRI study reported activity changes during the processing of the musical stimuli in limbic and para-limbic brain structures (amygdala, hippocampus, parahippocampal gyrus and temporal poles), which are well known to be crucially involved in the processing of emotion. Therefore, the present experiment aimed at investigating whether BOLD signal changes in these structures correlate with the $E_{\kappa}$ values of subjects (that is, with the cardiac signature obtained in Experiment 1). It was hypothesized that differences in the $E_{\kappa}$ values (taken to indicate differences in emotionality) are related to differences in brain activity in structures that are involved in the processing of emotion.

\section{Materials and methods, Experiment 3a}

\section{Subjects}

Seventeen healthy individuals without psychiatric disorders (age range 19-28 years, mean 24.6 years, nine females) participated in the experiment. All participants were right-handed, free of known chronic medical conditions, and were not taking any medication for at least 4 weeks prior to the study. Participants' range of $E_{\kappa}$ values was $0.04-1.45$, mean $=0.53, \mathrm{SD}=0.34$; three participants had low $E_{\kappa}$ values (below 0.4), nine participants had high $E_{\kappa}$ values (above 0.8 ) and five had intermediate $E_{\kappa}$ values (between 0.4 and $0.8)$.

Stimuli, procedure and MR scanning were identical to a previous study, and have been described in detail elsewhere (Koelsch et al., 2006). In brief, pleasant stimuli were eight excerpts of joyful instrumental dance-tunes from the last four centuries (all major-minor tonal music), recorded from commercially available CDs. Unpleasant stimuli were electronically manipulated counterparts of the original tunes (stimuli were processed using Syntrillium Cool Edit Pro 2.0). For each pleasant stimulus, a new sound-file was created in which the original (pleasant) excerpt was recorded simultaneously with two pitch-shifted versions of the same excerpt, the pitch-shifted versions being one semi tone above and a tritone below the original pitch (samples of the stimuli are available at http://www.stefan-koelsch.de). Pleasant and unpleasant excerpts were presented in alteration, the duration of excerpts was 45-60 s (mean duration $55 \mathrm{~s}$ ). All excerpts were presented twice during the functional measurements to increase the signal-to-noise ratio. Excerpts were separated by a 3 -s interval with no stimulation. During this interval, participants indicated on a fivepoint scale how (un)pleasant they felt by pressing response buttons according to a five-point scale (with -2 corresponding to very unpleasant, 0 neutral and +2 very pleasant). Participants were instructed to listen carefully to the music and to tap the metre of the music with their right index finger. This task allowed to control if participants paid attention not only to the consonant, but also to the dissonant stimuli.

Scanning was performed on a $3 \mathrm{~T}$ Bruker Medspec 30/100 spectrometer. Nine axial slices $(19.2 \mathrm{~cm}$ FOV, $64 \times 64$ matrix, $5 \mathrm{~mm}$ slice thickness, $2 \mathrm{~mm}$ interslice gap), parallel to the AC-PC plane defined by the anterior and the posterior commissure (five slices were above this AC-PC plane), using a single shot, gradient-recalled EPI (flip angle $90^{\circ}, \mathrm{TR}=3000 \mathrm{~ms}$ ) were acquired. Two functional sessions, each 360 time points, were run. Each time point sampled over the nine slices. The relatively low number of slices and the relatively long TR was used to reduce the scanner noise per time unit (whole brain coverage will be used in Experiment 3b). fMRI data were processed using the software package LIPSIA (Lohmann et al., 2001). After slicetime-, motion- and baseline-correction (filtering with cutoff frequency of $1 / 132 \mathrm{~Hz}$ ), and applying a spatial Gaussian filter with $5.6 \mathrm{~mm}$ FWHM, functional images were registered into stereotactic space. Statistical evaluation for each subject was based on a least squares estimation using the general linear model for serially autocorrelated observations. The design matrix was generated with a boxcar (square wave) function. Observation data and design matrix were convolved using a Gaussian kernel of dispersion of $4 \mathrm{~s}$ FWHM. The output image contains the computed beta-values, which are an estimate for the slope of the regression. These images were evaluated for each subject using a linear contrast. To test the correlation between contrast values and ECG indices, a second-level statistical analysis based on the aligned contrast images was performed using $E_{\mathrm{\kappa}}$ values of subjects as a regressor.

\section{Results, Experiment 3a}

\section{Behavioural data}

The average rating for the consonant stimuli was 1.2 (on a five-point scale ranging from -2 to +2 ), for the dissonant stimuli -0.7 (the difference between ratings was significant, $P<0.0001$, two-tailed $t$ test). The ratings performed by the participants did not correlate with the $E_{\mathrm{\kappa}}$ values $(P>0.9)$.

\section{fMRI data}

For each participant, a contrast image was computed for the contrast unpleasant vs pleasant music. The contrast images were then correlated within a second-level analysis with the individual $E_{\mathrm{\kappa}}$ values of participants using voxel-wise correlations (Fig. 3A, see also Table 1). Results indicated significant correlations between the $E_{\mathrm{\kappa}}$ values (measured prior to the functional imaging session) and the BOLD responses elicited by the musical stimuli in the left amygdala $(r=0.68, P<0.0001)$, and in the right hippocampal formation ( $r=0.42, P<0.0005)$ : The higher the $E_{\kappa}$ values, the stronger were the BOLD signal changes within the amygdala and the hippocampal formation in response to the unpleasant (compared with the pleasant) music.

\section{Experiment 3b}

To replicate the findings of Experiment 3a, a second fMRI experiment was conducted with an independent group of subjects, using an experimental protocol similar to that of Experiment 3a, but with whole-brain coverage and without interference between the auditory stimuli and the scanner noise. Moreover, we directly compared two groups of equal sample size: group 1 consisted of 12 subjects with lower $E_{\kappa}$ values (below 0.45 ), group 2 of 12 subjects with higher $E_{\kappa}$ values (above 0.55).

\section{Materials and methods, Experiment $3 b$}

\section{Subjects}

Twenty-four healthy individuals without psychiatric disorders (age range 22-33 years, mean age 25.3 years, 12 females) participated in the second fMRI experiment. All participants were right-handed, free of known chronic medical conditions, and were not taking any 

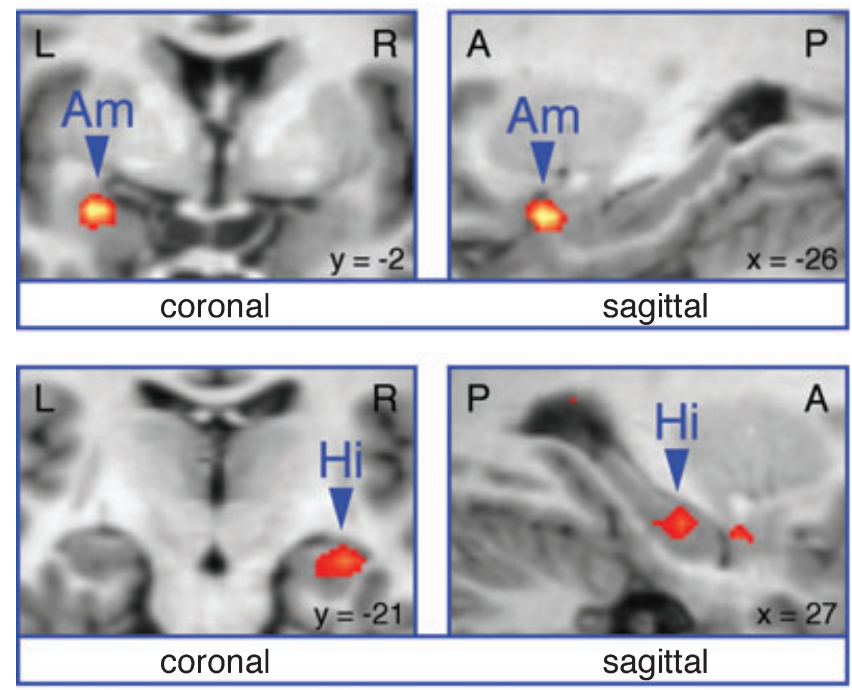

2.6 4.02
B Experiment 3b
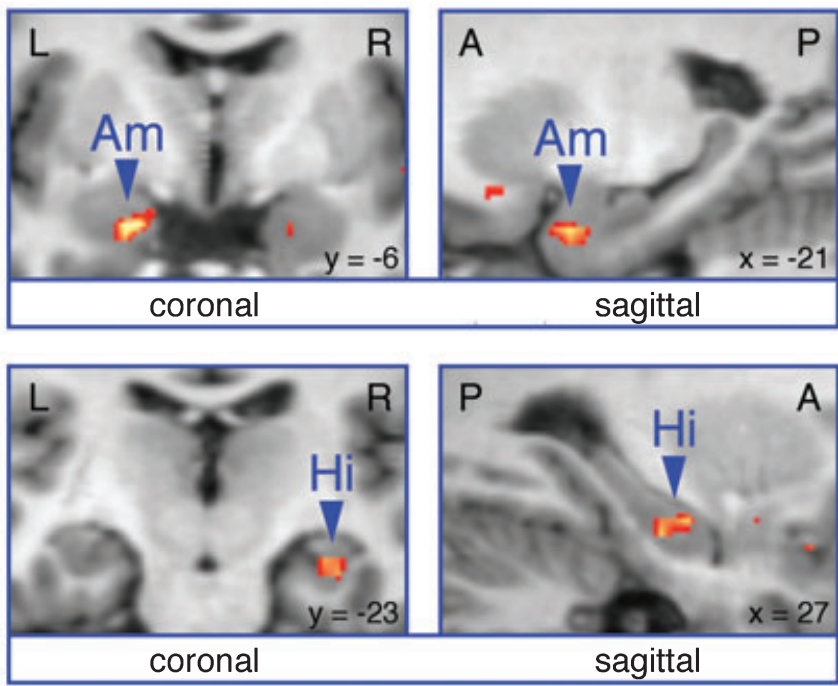

2.6

3.24

FIG. 3. Results of fMRI experiments. (A) In Experiment 3a, contrast images were first computed for each participant ( $n=17$, contrast: unpleasant vs pleasant), and then correlated with individual $E_{\mathrm{K}}$ values using voxel-wise correlations (second-level analysis; the $Z$-value threshold for depiction of statistical significance of voxels was 2.6, corresponding to $P<0.005$ ). Significant correlations between BOLD signal changes and $E_{\mathrm{\kappa}}$ values were found in the left amygdala (Am, upper panel), and the right hippocampal formation (Hi, lower panel). (B) In Experiment $3 \mathrm{~b}$, a group of subjects with low $E_{\mathrm{\kappa}}$ values (below 0.45 ) was compared with a group of subjects with higher $E_{\mathrm{\kappa}}$ values (above 0.55 ). The individual contrast images ( $n=24$, contrast: unpleasant vs pleasant) were compared between groups using voxel-wise $t$-tests (second level analysis; the $Z$-value threshold for depiction of statistical significance of voxels was 2.6 , corresponding to $P<0.005$ ). As in Experiment $3 \mathrm{a}$, differences in haemodynamic responses were found as a function of the $E_{\mathrm{K}}$ values in the left amygdala (Am, upper panel), and the right hippocampal formation (Hi, lower panel).

TABle 1. Activations from the statistical parametric maps presented in Fig. 3

\begin{tabular}{lrrrlll}
\hline & \multicolumn{2}{l}{ Talairach coordinates } & & \\
\cline { 2 - 4 } Brain region & $x$ & $y$ & $z$ & $\begin{array}{l}\text { Maximum } \\
Z \text {-value }\end{array}$ & $\begin{array}{l}\text { Cluster } \\
\text { size }\left(\mathrm{mm}^{3}\right)\end{array}$ \\
\hline Experiment 3a & & & & & \\
$\quad$ Left amygdala & -26 & -2 & -12 & 4.02 & 216 \\
$\quad$ Right hippocampus & 27 & -21 & -12 & 3.40 & 81 \\
$\quad \begin{array}{l}\text { Experiment 3b } \\
\quad \text { Left amygdala }\end{array}$ & -21 & -6 & -15 & 3.13 & 135 \\
$\quad$ Right hippocampus & 27 & -23 & -12 & 3.24 & 270 \\
\hline
\end{tabular}

The $Z$-value threshold for statistical significance of voxels was 3.09 $(P<0.001$, uncorrected). Coordinates refer to standard stereotactic space (Talairach \& Tournoux, 1998).

medication for at least 4 weeks prior to the studies. Participants were divided into two groups, each group consisting of six males and six females. The range of $E_{\mathrm{\kappa}}$ values was $0.03-1.61 \quad(\mathrm{M}=0.60$, $\mathrm{SD}=0.53$ ), the highest $E_{\kappa}$ value in group 1 was 0.45 , the lowest $E_{\kappa}$ value in group 2 was 0.55 .

Stimuli were similar to those used in the first fMRI experiment (excerpts of joyful instrumental tunes and their electronically manipulated, permanently dissonant counterparts). In this experiment, both types of stimuli were also played backwards (this increased the degree of unpleasantness of permanently dissonant excerpts). In contrast to Experiment $3 \mathrm{a}$ (where continuous scanning was applied), the fMRI data of Experiment $3 \mathrm{~b}$ were recorded with a sparse temporal sampling design (TR $=12 \mathrm{~s}$, clustered volume acquisition of $2.1 \mathrm{~s}$ duration). Such designs allow the presentation of auditory stimuli in the absence

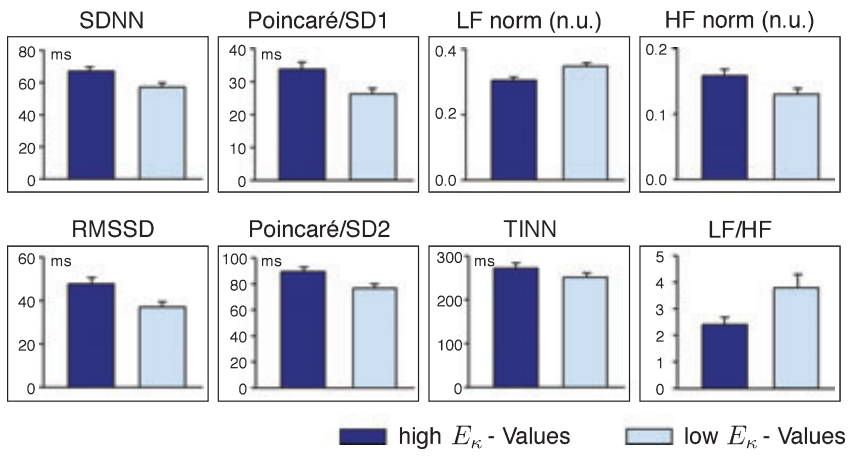

FIG. 4. Results of 35 min HRV measurements, separately for subjects with higher $E_{\mathrm{\kappa}}$ values ( $>0.45$, corresponding to higher emotionality, dark blue bars) and lower $E_{\kappa}$ values $(<0.45$, corresponding to lower emotionality, light blue bars; error bars indicate standard errors of means). In both the time and the frequency domain, clear group differences were observed, indicating significant differences in tonic cardiac autonomic activity between groups. LF norm (n.u.): low-frequency power in normalized units $(0.04-0.15 \mathrm{~Hz})$; HF norm (n.u.): high-frequency power in normalized units $(0.15-0.4 \mathrm{~Hz})$; LF/HF: ratio of LF to $\mathrm{HF}$.

of fMRI scanner noise between two consecutive volume acquisitions (Hall et al., 1999), so that the scanner noise does not interfere with the auditory stimulation. This design had the additional advantage that whole brain coverage (with 24 slices) could be used without interfering with the auditory stimulation. To provide for a broad sampling of the BOLD response, temporal jittering was applied by presenting stimuli at 10 different time points relative to image acquisition (i.e. relative to the trial onset). Onsets (in s) were 2, 2.7, 
3.4, 4.1, 4.8, 5.6, 6.3, 7.0, 7.7 and 8.4, resulting in durations of stimuli between 3.6 and $10 \mathrm{~s}$. At the end of each trial (i.e. during the scanning), participants had to indicate how the music had influenced their emotional state (positively or negatively) on a six-point scale.

Scanning was performed on a $3 \mathrm{~T}$ Magnetom Trio (Siemens, Erlangen, Germany). Twenty-four axial slices (parallel to the AC-PC plane) were acquired, using a gradient-echo EPI sequence ( $\mathrm{TE}=30 \mathrm{~ms}$, flip angle $90^{\circ}, \mathrm{TR}=12 \mathrm{~s}$, acquisition bandwidth $100 \mathrm{kHz}$ ). The acquired matrix was $64 \times 64$ and the FOV $19.2 \mathrm{~cm}$, resulting in an in-plane resolution of $3 \mathrm{~mm}^{2}$. The slice thickness was $4 \mathrm{~mm}$ and the interslice gap $1 \mathrm{~mm}$. Analysis of the fMRI data was identical to Experiment 1, except that a second-level analysis based on the aligned contrast images was performed for two groups (groups were assigned based on $E_{\kappa}$ values): this second-level analysis consisted of a voxel-wise two-sample $t$-test across the contrast images of both groups.

\section{Results, Experiment $3 b$}

As in Experiment 3a, contrast images were computed for the contrast unpleasant vs pleasant, separately for each participant. Then, these contrast images were compared between groups within a second-level analysis using voxel-wise $t$-tests (Fig. 3B and Table 1). Results replicated the findings of the first experiment. Activity changes in response to the unpleasant (compared with the pleasant) music differed between groups in both the left amygdala and the right hippocampal formation (see Table 1 for statistical values). Again, BOLD signal changes within these structures were stronger in subjects with high $E_{\kappa}$ values than in subjects with low $E_{\kappa}$ values.

\section{Discussion, Experiments $3 a$ and $3 b$}

In both Experiments $3 \mathrm{a}$ and $3 \mathrm{~b}$, activity changes in the amygdala and the hippocampal formation were stronger in subjects with high $E_{\kappa}$ values (corresponding to a higher emotionality) than in subjects with low $E_{\mathrm{\kappa}}$ values (corresponding to a lower emotionality). The amygdala is a central structure of the limbic system, and well known to be crucially involved in emotional processing (Mega et al., 1997). Patients with amygdala removal or amygdala lesions show impaired appraisal of auditory signals with emotional valence, and functional imaging studies have shown that the amygdala is involved in the detection and generation of emotion (Gosselin et al., 2005; Phelps \& LeDoux, 2005). Moreover, activity changes in the amygdala have previously been shown to correlate with personality parameters related to emotion (such as extraversion and neuroticism; Canli et al., 2002). The hippocampus proper (which is also considered a limbic structure) is densely interconnected with the amygdala, and critically involved in a number of emotion-related brain functions such as encoding and retrieval of stimuli with emotional significance, modulation of defensive behaviour, affective state regulation, attention and motivation (Siebel \& Winkler, 1996; Mega et al., 1997). The hippocampus has been shown to be sensitive to audiogenic stressors, and the hippocampal region that was more strongly activated in subjects with higher emotionality (Fig. 3) has been shown to be damaged in patients with severe post-traumatic stress disorder (Bremner, 1999), highlighting the involvement of the hippocampus in emotional processing.

Our findings, thus, show that a specific cardiac signature predicts the amount of activity changes in brain structures implicated in the processing of emotion: higher $E_{\mathrm{\kappa}}$ values correspond to stronger activity changes in those structures, whereas lower $E_{\kappa}$ values correspond to weak activity changes in those structures (possible relations between $E_{\mathrm{K}}$ values, HR and BOLD responses will be touched upon in Experiment 4). Note that neither musical nor any other stimuli were presented during the ECG measurements (ECG data were acquired at least 1 day prior to the functional imaging experiments). Thus, the ECG signature of an individual's resting ECG predicted the emotional brain activity elicited later on in the functional imaging experiments.

These results show a close connection between limbic structures and the heart in healthy humans. Previous research showed that cardiovascular indices such as HR, HRV or blood pressure inform about processes of the autonomic or hormonal system. Our data reveal that cardiac signals also provide information about neurophysiological processes located beyond the level of autonomic control, namely on the level of neurophysiological processes linked to individual characteristics of emotional processing. This implies that the interactions between the brain and the heart are more complex than previously believed, calling for a better understanding of these interactions so that they can be taken into account when investigating the aetiology of cardiovascular disease.

\section{Experiment 4: relations between the cardiac signature and autonomic balance}

To further test the hypothesis of a cardiac signature of emotionality, we correlated $E_{\mathrm{\kappa}}$ values with parameters of both the time and the frequency domain of $\mathrm{HRV}$, an established tool to assess cardiac autonomic nervous system (ANS) activity (Malik et al., 1996). The HR continuously varies in relation to breathing, blood pressure, as well as physical, mental or emotional activity, and numerous other factors (Stein et al., 1994). During inhalation, for example, the HR slightly accelerates, while it decelerates during exhalation. This variability of the HR has been shown to provide important information about autonomic balance (i.e. balance or predominance of either the sympathetic or the parasympathetic branch of the ANS), and autonomic tone (i.e. strength of sympathetic and parasympathetic activation).

Note that emotional activity always has effects on the activity of the ANS, and that ANS centres located in the brain stem and in the hypothalamus are densely interconnected with both the amygdala and the hippocampus (which had shown higher BOLD responses in subjects with higher emotionality). Especially the amygdala (in concert with insular and orbitofrontal cortices) exerts direct influence on neurons involved in autonomic cardiovascular control. Animal data showed that the amygdala directly projects to autonomic neurons in the hypothalamus, as well as to brainstem neurons located in the parabrachial nucleus, the nucleus of the solitary tract and the dorsal nucleus of the vagus (Cechetto, 2004). Site-dependent electrical stimulation of the amygdala modulates HR (as well as blood pressure), and microinjections of glutamate in the amygdala increase HR (Gelsema et al., 1987; Iwata et al., 1987). Because of the close relation between emotion and (cardiac) autonomic activity, it was expected that differences in emotionality - as indicated by the $E_{\mathrm{\kappa}}$ values - are also reflected in differences in HRV.

\section{Materials and methods, Experiment 4}

Subjects

Data from 66 healthy individuals without psychiatric disorders (age range 19-34 years, mean 24.8 years, 32 females) entered the statistical evaluation (participants with extremely high SDNN [>100] or 
extremely low RMSSD $[<20]$ had been excluded from further analysis; for explanation of SDNN and RMSSD, see following paragraph). All participants had normal blood pressure (below $130 / 85 \mathrm{mmHg}$ ), a body mass index ranging from 18 to 26 , and reported to be free of any known medical diseases.

HRV measurements were performed using standard procedures according to the guidelines of the Task Force of the European Society of Cardiology and the North American Society of Pacing and Electrophysiology (Malik et al., 1996), except that HRV recordings were performed while participants listened to the pleasant and unpleasant musical stimuli also presented in the fMRI experiments. We computed the SDNN (standard deviation of the time intervals between consecutive heart beats, also denoted as $\mathrm{NN}$ intervals), the RMSSD (square root of the mean squared differences of successive $\mathrm{NN}$ intervals), and the TINN (triangular interpolation of $\mathrm{NN}$ intervals histogram), which are prominent time domain measures of the HRV (Stein et al., 1994; Malik et al., 1996). In addition to these parameters, frequency domain measures were computed (i.e. power spectral density analyses), which provide further information about autonomic balance. A low-frequency component (LF, taken to reflect sympathetic or perhaps both sympathetic and parasympathetic effects; Malik et al., 1996) was computed for the frequency range from 0.04 to $0.15 \mathrm{~Hz}$, and a high-frequency component (HF, taken to reflect mainly parasympathetic effects; Malik et al., 1996) was computed for the range from 0.15 to $0.4 \mathrm{~Hz}$. Low- and high-frequency components were also calculated in normalized units, which particularly reflect the controlled and balanced behaviour of the two ANS branches (Malik et al., 1996). Finally, the SD of the instantaneous beat-to-beat variability (Poincaré SD1), and the SD of the continuous long-term R$\mathrm{R}$ intervals (Poincaré SD2) were calculated (these measures are taken to reflect parasympathetic nervous system activity; Kamen et al., 1997; Lerma et al., 2003). HRV measures were computed using the BIOSIG toolbox (Schlögl et al., 2007).

\section{Results and discussion, Experiment 4}

Subjects were divided into two groups based on a median split of $E_{\kappa}$ values (median: 0.45). One-sided, two-sample $t$-tests on the HRV parameters indicated clear differences in time and frequency domain measures, as well as for the geometric measures of the HRV between groups (Fig. 4, see Table 2 for results of statistical tests). This

TABLE 2. Results of the 35 min HRV measurements

\begin{tabular}{lccc}
\hline Parameter & Group 1 (high) & Group 2 (low) & $P$-value \\
\hline SDNN (ms) & $67.1 \pm 2.9$ & $57.3 \pm 2.6$ & 0.01 \\
RMSSD (ms) & $47.7 \pm 3.0$ & $37.1 \pm 2.4$ & 0.01 \\
TINN (ms) & $272.3 \pm 11.6$ & $252.3 \pm 9.2$ & 0.05 \\
Poincare SD1 (ms) & $33.8 \pm 2.1$ & $26.3 \pm 1.7$ & 0.01 \\
Poincare SD2 (ms) & $89.2 \pm 3.7$ & $76.5 \pm 3.3$ & 0.02 \\
LF (ms ${ }^{2}$ ) & $1169 \pm 148$ & $1079 \pm 122$ & n.s. \\
HF (ms ${ }^{2}$ ) & $578 \pm 60$ & $404 \pm 53$ & 0.05 \\
LF norm (n.u.) & $0.304 \pm 0.01$ & $0.347 \pm 0.01$ & 0.01 \\
HF norm (n.u.) & $0.157 \pm 0.01$ & $0.129 \pm 0.01$ & 0.05 \\
LF/HF & $2.4 \pm 0.28$ & $3.8 \pm 0.49$ & 0.02 \\
\hline
\end{tabular}

Means (and standard errors of means) are indicated separately for subjects with higher $E_{\mathrm{\kappa}}$ values ( $>0.45$, corresponding to higher emotionality, group 1) and lower $E_{\mathrm{\kappa}}$ values $(<0.45$, corresponding to lower emotionality, group 2$)$. The outer right column provides $P$-values of two-tailed, independent samples $t$-tests. Abbreviations as in Fig. 4. demonstrates a relation between the $E_{\kappa}$ values and the autonomic activity of individuals.

Notably, compared with subjects with higher $E_{\kappa}$ values (corresponding to higher emotionality), subjects with low $E_{\kappa}$ values exhibited significantly lower values of time domain HRV parameters. Moreover, the frequency domain parameters revealed a significantly lower HF component, and an increased LF/HF ratio in subjects with low $E_{\kappa}$ values. This pattern clearly reproduces the HRV pattern previously reported in studies investigating patients with major depression and pathologic anxiety (Yeragani et al., 1993, 2000, 2002; Friedman \& Thayer, 1998), that is, with disorders that are characterized by frequent phases of absence of positive emotions. These studies thus suggest that decreased HRV (along with a low HF component and increased $\mathrm{LF} / \mathrm{HF}$ ratio) is related to decreased positive emotionality. The finding that this HRV pattern is also observed in individuals with low $E_{\kappa}$ values hence supports the assumption that lower $E_{\mathrm{\kappa}}$ values are related to a reduction of tender positive emotionality. However, increased negative emotionality in individuals with pathologic anxiety and depression does not necessarily mean that such individuals have a persistent lack of tender positive emotions. Thus, it remains to be investigated whether there is an association between anxiety or depression and lower $E_{\kappa}$ values.

As in Experiment 1, no correlations were found between $E_{\kappa}$ values on the one hand, and body mass index, body height or body weight on the other $(r<0.2$ and $P>0.15$ in all tests; this was to be expected because only amplitude ratios are considered when calculating $E_{\mathrm{\kappa}}$, see Experiment 1). Likewise, no correlations between $E_{\mathrm{\kappa}}$ values and the electrical axis of the heart (Engblom et al., 2005) or blood pressure were indicated $(r<0.2$ and $P>0.15$ in all tests). This confirms that the $E_{\kappa}$ values are not related to body size, the heart's electrical axis or blood pressure. Notably, no differences were found in HR between groups (mean HR was 71.2 in the group with low, and 71.4 in the group with high $E_{\kappa}$ values), rendering it unlikely that differences in HR account for the group-specific BOLD responses in Experiments $3 \mathrm{a}$ and $3 b$.

\section{General discussion}

Our results reveal a clear relation between emotional personality and ECG amplitude ratios. So far, modulations of $\mathrm{T}$ - (and P-) wave amplitudes have been associated with vagal tone. By showing that ratios of ECG amplitudes vary as a function of an individual's emotional personality, our results demonstrate that amplitudes of the ECG bear considerably more information than previously believed. This discovery has several important implications.

\section{Interactions between the brain and the heart}

The present data demonstrate that interactions between the brain and the heart are not only involved in the regulation of vagal tone and cardiovascular reflexes (Armour \& Ardell, 2004), but that these interactions also express intricate connections between personality and biology. Given that heart disease is one of the major health problems in both the USA and Europe, our findings call for a much better understanding of these connections so that they can be taken into account when investigating the aetiology of cardiovascular disease. Moreover, although we did not show this directly, our results render it likely that interactions between the brain and the heart (and thus heart function and possible health risks) may be influenced by psychological treatment. 


\section{A physiological measure of emotionality}

The relations between $E_{\kappa}$ values and TAS-1 scores (Experiment 1), averaged emotionality ratings (Experiment 2 ), brain activity reflecting emotional processing (Experiments $3 \mathrm{a}$ and $3 \mathrm{~b}$ ), and (cardiac) autonomic activity (Experiment 4 ) indicate that the $E_{\kappa}$ values can be used for the identification of interindividual differences in emotionality. It is possible that the $E_{\mathrm{\kappa}}$ values are also affected by emotional stress or other psychological variables (this remains to be further specified), but the high correlation between test and re-test values suggests that the $E_{\mathrm{\kappa}}$ values mainly vary as a function of emotional personality, rather than of emotional states (note that this does not exclude the possibility that an individual's emotional personality can change and that such changes can lead to different $E_{\mathrm{\kappa}}$ values).

Previous research on the biological basis of personality has identified some correlations between personality traits and biological measures (such as HR, HRV, cortical activation levels, skin conductivity and hormone levels, see Introduction), but literature on the assessment of personality with physiological measures is surprisingly scant. Thus, psychological and medical research on personality differences has so far mainly relied on subjective measures of personality, such as questionnaires and interviews (Davidson et al., 2003). Compared with such measures, the cardiac signature reported here has the advantage that it is not influenced by subjective bias, although it may be affected by factors unrelated to personality. Subjective personality instruments, on the other hand, are designed to specifically examine aspects of personality. Thus, the use of $E_{\kappa}$ values, perhaps in conjunction with standardized self-report and observational personality indices, opens new perspectives for a more accurate personality assessment, and thus for clinical investigations on relations between emotional dysbalance and organic disease, as well as for a preclinical diagnosis of emotional dysbalance and related health risks.

With respect to the diagnostic potential of the $E_{\kappa}$ values, emotional impairment associated with persistent lack of tender positive feelings - previously described as hyperathymia maxima (Siebel, 1994; Siebel \& Winkler, 1996) - appears to be highly correlated with low $E_{\kappa}$ values. The correlation between $E_{\kappa}$ values and TAS-1 scores supports this assumption. Thus, future studies on biological correlates of emotion could make use of the $E_{\kappa}$ values to screen out subjects with reduced emotional responses, and hence increase the signal-to-noise ratio of experimental data.

Notably, the relation between reduced $E_{\kappa}$ values and reduced HRV would suggest that individuals with lower $E_{\mathrm{\kappa}}$ values have an increased risk for cardiac mortality, particularly after myocardial infarction or congestive heart failure (Hamaad et al., 2004). It remains to be specified whether extremely high $E_{\kappa}$ values are also related with health risks.

\section{Possible modulators of ECG amplitude ratios}

The exact mechanisms that lead to the differences in the observed cardiac signatures remain to be specified. According to our data, $\mathrm{E}_{\mathrm{\kappa}}$ values are not simply dependent on blood pressure, body size or electrical axis of the heart (no significant correlations were indicated between these factors and $E_{\kappa}$ values). One possible explanation for interindividual differences in $E_{\mathrm{\kappa}}$ values is that differences in the cardiac amplitude signatures are related to differences in lateralization of autonomic control. Individuals with low $E_{\kappa}$ values showed a greater autonomic imbalance (as indicated, e.g. by the increased ratio of lowfrequency to high-frequency components of the HRV), and previous work has shown that autonomic imbalance is associated with lateralization of central autonomic drive (Craig, 2005). Central autonomic drive is channelled ipsilaterally to the heart, where strongly lateralized autonomic drive leads to a disruption of signal transduction mechanisms on the anterior and posterior heart surface (Critchley et al., 2005). The right cardiac nerve innervates the anterior surface of the heart (including the sinoatrial node, the atrioventricular node, and the anterior surfaces of the right and left ventricles), whereas the left cardiac nerve innervates the posterolateral surface of the heart (including the atrioventricular node and the posterior surfaces of the right and left ventricles; Lane, \& Schwartz, 1987). Thus, it is likely that the autonomic imbalance of individuals with low $E_{\kappa}$ values results in a left-right imbalance in autonomic drive across the surface of the heart, which disrupts the electrophysiological homogeneity of ventricular (re)polarization (Critchley et al., 2005).

Previous research also suggested that negative feelings, such as discontent, anger and competitiveness, are associated with autonomic functions involved in signal transduction mechanisms that might lead to left ventricular hypertrophy (Agewall et al., 1996), and it is possible that such a morphological factor accounts at least partly for the ECG pattern described in the present study. However, measurements of $\mathrm{N}$-terminal-pro brain natriuretic peptide (NT-pro BNP; Hall, 2004) obtained from 16 individuals did not yield a correlation between $E_{\kappa}$ values and NT-pro BNP serum concentrations. Because NT-pro BNP is associated with left ventricular hypertrophy, and correlates with left ventricular mass (e.g. Luchner et al., 2002), this suggests that gross morphological differences between the hearts of individuals with higher and lower $E_{\kappa}$ values are highly unlikely, at least with respect to left ventricular mass.

[Rest ECGs and blood samples were obtained from 16 healthy individuals without psychiatric disorder (age range 21-31 years, mean age 26.3 years, eight females), all individuals had normal blood pressure (below 130/85 $\mathrm{mmHg}$ ), and a body mass index between 18 and 27. Half of the subjects had $E_{\kappa}$ values below 0.45 and the other half had $E_{\kappa}$ values above 0.55 . The range of NT-pro BNP serum concentrations was $8-69 \mathrm{pg} / \mathrm{mL}$, that is, for all subjects, NT-pro BNP serum concentrations were within the norm range. A two-tailed, independent samples $t$-test comparing the NT-pro BNP values between groups did not indicate a difference $(P>0.90)$.]

Finally, it is important to note that the manifold effects of emotional activity on autonomic activity, hormone and electrolyte regulation modulate the regulatory role that some neurons in intrinsic cardiac and intrathoracic extracardiac ganglia play in the control of regional cardiac function (Armour \& Ardell, 2004). It is conceivable that such modulations also lead to specific signatures in the amplitude pattern of the ECG in individuals with persistent emotional dysbalance. The exact nature of such modulations, however, remains to be investigated.

\section{Conclusions}

Our results reveal strong correlations between emotional personality and ECG amplitude ratios. This indicates that ECG amplitudes contain more information than previously believed, and that connections between the brain and the heart - which mediate regulation of cardiac autonomic activity and cardiovascular reflexes - are considerably influenced by an individual's emotional personality, even in nonclinical populations. The cardiac emotionality measure described in our study opens the perspective for a more accurate assessment of personality using ECG measures.

\section{Acknowledgements}

We thank Claudia Wiebigke, Christian Girbardt and Siri Kamp for their help in data acquisition and analysis, as well as D.Y.v. Cramon, Johannes Greten, Thomas C. Gunter, Sonja Kotz and Niko Steinbeis for valuable comments. The 
study was supported in part by the German Research Foundation (Deutsche Forschungsgemeinschaft) through grant KO 2266/2-1.

\section{Abbreviations}

ANS, autonomic nervous system; BOLD, blood oxygen level-dependent; ECG, electrocardiography; fMRI, functional magnetic resonance imaging; HF, high frequency component of the HRV; HR, heart rate; HRV, heart rate variability; LF, low frequency component of the HRV; NEO PI-R, Revised NeuroticismExtraversion-Openness Personality Inventory; NT-pro BNP, N-terminal-pro brain natriuretic peptide; TAS, Toronto Alexithymia Scale.

\section{References}

Ader, R. (2006) Psychoneuroimmunology, 4th Edn. Academic Press, San Diego, CA.

Adler, N. \& Matthews, K. (1994) Health psychology - why do some people get sick and some stay well. Ann. Rev. Psych., 45, 229-259.

Agewall, S., Wikstrand, J., Dahlöf, C. \& Fagerberg, B. (1996) Negative feelings (discontent) predict progress of intima-media thickness of the common carotid artery in treated hypertensive men at high cardiovascular risk. Am. J. Hypertens., 9, 545-550.

Andreasen, N.C. (1982) Negative symptoms in schizophrenia. Definition and reliability. Arch. Gen. Psychiatry, 39, 784-788.

Antaki, C., Hewstone, M., Stroebe, W., Codol, J. \& Stephenson, G.M. (1988) Introduction to Social Psychology. Blackwell Science, Oxford.

Armour, J.A. \& Ardell, J.L. (2004) Basic and Clinical Neurocardiology. Oxford University Press, New York, NY.

Biondi, M. \& Picardi, A. (1999) Psychological stress and neuroendocrine function in humans: the last two decades of research. Psychoth. Psychosoma, 68, 114-150.

Bremner, J. (1999) Does stress damage the brain? Biol. Psychiatry, 45, 797805.

Cacioppo, J.T., Berntson, G.G., Larsen, J.T., Poehlmann, K.M. \& Ito, T.A. (2000) The psychophysiology of emotion. In Lewis, M. \& Haviland-Jones, J.M. (Eds), The Handbook of Emotion, 2nd Edn. Guilford Press, New York, pp. 173-191.

Canli, T., Sivers, H., Whitfield, S.L., Gotlib, I.H. \& Gabrieli, J.D. (2002) Amygdala response to happy faces as a function of extraversion. Science, 296, 2191.

Carney, R.M., Blumenthal, J.A., Stein, P.K., Watkins, L., Catellier, D., Berkman, L.F., Czajkowski, S.M., O’Connor, C., Stone, P.H. \& Freedland, K.E. (2001) Depression, heart rate variability, and acute myocardial infarction. Circulation, 104, 2024-2028.

Carver, C.S. \& Miller, C.J. (2006) Relations of serotonin function to personality: current views and a key methodological issue. Psychiat. Res., 144, 1-15.

Cechetto, D.F. (2004) Forebrain control of healthy and diseased hearts. In Armour, J.A. \& Ardell, J.L. (Eds), Basic and Clinical Neurocardiology. Oxford University Press, New York, NY, pp. 220-251.

Costa, P.T. \& McCrae, R.R. (1992) NEO PI-R. Professional Manual. Psychological Assessment Resources, Odessa, FL.

Craig, B. (2005) Forebrain emotional asymmetry: a neuroanatomical basis? Trends Cogn. Sci., 9, 566-571.

Critchley, H.D., Taggart, P., Sutton, P.M., Holdright, D.R., Batchvarov, V., Hnatkova, K., Malik, M. \& Dolan, R.J. (2005) Mental stress and sudden cardiac death: asymmetric midbrain activity as a linking mechanism. Brain, 128, 75-85.

Davidson, R.J., Scherer, K.R. \& Goldsmith, H. (2003) Handbook of Affective Sciences. Oxford University Press, New York, NY.

Engblom, H., Foster, J.E., Martin, T.N., Groenning, B., Pahlm, O., Dargie, H.J., Wagner, G.S. \& Arheden, H. (2005) The relationship between electrical axis by 12-lead electrocardiogram and anatomical axis of the heart by cardiac magnetic resonance in healthy subjects. Am. Heart J., 150, 507-512.

Eysenck, H.J. (1990) Biological dimensions of personality. In Pervin, L.A. (Ed.), Handbook of Personality: Theory and Research. Guilford Press, New York, pp. 244-276.

Friedman, B.H. \& Thayer, J.F. (1998) Autonomic balance revisited: panic anxiety and heart rate variability. J. Psychosom. Res., 44, 133-151.

Gelsema, A.J., McKitrick, D.J. \& Calaresu, F.R. (1987) Cardiovascular responses to chemical and electrical stimulation of amygdala in rats. Am. J. Physiol., 253, 712-718.
Gosselin, N., Peretz, I., Noulhiane, M., Hasboun, D., Beckett, C., Baulac, M. \& Samson, S. (2005) Impaired recognition of scary music following unilateral temporal lobe excision. Brain, 128, 628-640.

Griffiths, J., Ravindran, A.V., Merali, Z. \& Anisman, H. (2000) Dysthymia: a review of pharmacological and behavioral factors. Mol. Psychiatry, 5, 242261.

Hall, C. (2004) Essential biochemistry and physiology of (NT-pro) BNP. Eur. J. Heart Failure, 6, 257-260.

Hall, D.A., Haggard, M.P., Akeroyd, M.A., Palmer, A.R., Summerfield, A.Q., Elliott, M.R., Gurney, E.M. \& Bowtell, R.W. (1999) 'Sparse' temporal sampling in auditory fMRI. Hum. Brain Mapp., 7, 213-223.

Hamaad, A., Lip, G.Y.H. \& MacFayden, R.J. (2004) Heart rate variability estimates of autonomic tone: relationship to mapping pathological and procedural stress responses in coronary disease. Ann. Med., 36, 448-461.

Haviland, M.G., Shaw, D.G., Cummings, M.A. \& MacMurray, J.P. (1988) Alexithymia: subscales and relationship to depression. Psychother. Psychosom., 50, 164-170.

Hollander, E. \& Evers, M. (2001) New developments in impulsivity. Lancet, 358, 949-950.

Iwata, J., Chida, K. \& LeDoux. J.E. (1987) Cardiovascular responses elicited by stimulation of neurons in the central amygdaloid nucleus in awake but not anesthetized rats resemble conditioned emotional responses. Brain Res., 18, $183-188$.

Joynt, K., Whellan, D.J. \& O'Connor, C.M. (2004) Why is depression bad for the failing heart? A review of the mechanistic relationship between depression and heart failure. J. Card. Fail., 10, 258-271.

Kamen, P.W., Krum, H. \& Tonkin, A.M. (1997) The correlation dimension of heart rate variability reflects cardiac autonomic activity. Ann. Noninvasive Electrocardiol., 2, 206-214.

Kiecolt-Glaser, J.K., McGuire, L., Robles, T.F. \& Glaser, R. (2002a) Psychoneuroimmunology and psychosomatic medicine: back to the future. Psychosom. Med., 64, 15-28.

Kiecolt-Glaser, J.K., McGuire, L., Robles, T.F. \& Glaser, R. (2002b) Emotions, morbidity, and mortality: new perspectives from psychoneuroimmunology. Ann. Rev. Psych., 53, 83-107.

Koelsch, S., Fritz, T., von Cramon, D.Y., Muller, K. \& Friederici, A.D. (2006) Investigating emotion with music: an fMRI study. Hum. Brain Mapp., 27, 329-350.

Kubzansky, L.D. \& Kawachi, I. (2000) Going to the heart of the matter: do negative emotions cause coronary heart disease? J. Psychosom. Res., 48, 323-337.

Kupfer, J., Brosig, B. \& Brähler, E. (2000) Überprüfung und Validierung der 26-Item Toronto-Alexithymie-Skala anhand einer repräsentativen Bevölkerungsstichprobe. Zeitschr. Psychosom. Med. Psychother, 46, 368-384.

Lane, R.D. \& Schwartz, G.E. (1987) Induction of lateralized sympathetic input to the heart by the CNS during emotional arousal: a possible neurophysiologic trigger of sudden cardiac death. Psychosom. Med., 49, 274-284.

Lerma, C., Infante, O., Pérez-Grovas, H. \& José, M.V. (2003) Poincaré plot indexes of heart rate variability capture dynamic adaptations after haemodialysis in chronic renal failure patients. Clin. Physiol. Funct. Imaging, 23, $72-80$.

Lesperance, F., Frasure-Smith, N. \& Talajic, M. (1996) Major depression before and after myocardial infarction: its nature and consequences. Psychosom. Med., 58, 99.

Lohmann, G., Müller, K., Bosch, V., Mentzel, H., Hessler, S., Chen, L., Zysset, S. $\&$ von Cramon, D.Y. (2001) Lipsia - a new software system for the evaluation of functional magnetic resonance images of the human brain. Comp. Med. Imaging Graph., 25, 449-457.

Lorber, M.F. (2004) Psychophysiology of aggression, psychopathy, and conduct problems: a meta-analysis. Psych. Bull., 130, 531-552.

Luchner, A., Hengstenberg, Chr, Löwel, H., Trawinski, J., Baumann, M., Riegger, G.A.J., Schunkert, H. \& Holmer, S. (2002) N-terminal pro-brain natriuretic peptide after myocardial infarction: a marker of cardio-renal function. Hypertension, 39, 99.

Lundh, L.-G. \& Simonsson-Sarnecki, M. (2001) Alexithymia, emotion, and somatic complaints. J. Personality, 69, 483-510.

MacMahon, K.M.A. \& Lip, G.Y.H. (2002) Psychological factors in heart failure - a review of the literature. Arch. Int. Med., 162, 509-516.

Malik, M. \& Task Force of the European Society of Cardiology and the North American Society of Pacing and Electrophysiology (1996) Heart rate variability: standards of measurement, physiological interpretation and clinical use. Eur. Heart J., 17, 354-381.

Martinez, J.P. \& Olmos, S. (2005) Methodological principles of T wave alternans analysis: a unified framework. IEEE Trans. Biomed. Engng, 52, 599-613. 
McLaughlin, N.B., Campbell, R.W.F. \& Murray, A. (1995) Influence of T wave amplitude on automatic QT measurement. Comp. Card., 777-780.

Mega, M.S., Cummings, J.L., Salloway, S. \& Malloy, P. (1997) The limbic system: an anatomic, phylogenetic, and clinical perspective. J. Neuropsychiatry Clin. Neurosci., 10, 315-330.

Netter, P. (2006) Dopamine challenge tests as an indicator of psychological traits. Hum. Psychopharmacol. Clin. Exp., 21, 91-99.

Pan, L., Contrada, R.J. \& Li, J.K.-J. (1992) Electrocardiographic T-wave amplitude as a non-invasive index of sympathetic myocardial activity: possible confounding effects of parasympathetic influence. Proc. IEEE Ann. Northeast, 123-124.

Pell, M.D. (2001) Influence of emotion and focus location on prosody in matched statements and questions. J. Acoust. Soc. Am., 109, 1668-1680.

Phelps, E.A. \& LeDoux, J.E. (2005) Contributions of the amygdala to emotion processing: from animal models to human behavior. Neuron, $\mathbf{4 8}$, 175-187.

Rozanski, A., Blumenthal, J.A. \& Kaplan, J. (1999) Impact of psychological factors on the pathogenesis of cardiovascular disease and implications for therapy. Circulation, 99, 2192-2217.

Rozanski, A. \& Kubzansky, L.D. (2005) Psychologic functioning and physical health: a paradigm of flexibility. Psychosom. Med., 67 (Suppl 1), S47-S53.

Rubinow, D.R. \& Schmidt, P.J. (1996) Androgens, brain, and behavior. Am. J. Psychiatry, 153, 974-984.

Russell, J.A. \& Fernández-Dols, J.M. (1997) The Psychology of Facial Expression. Cambridge University Press, New York.

Schlögl, A., Brunner, C., Scherer, R. \& Glatz, A. (2007) BioSig - an open source software library for BCI research. In Dornhege, G., Millan, J.R., Hinterberger, T., McFarland, D.J. \& Müller, K.-R. (Eds), Towards BrainComputer Interfacing. MIT Press, Cambridge, Massachusetts, pp. 347-358. [See also http://biosig.sf.net]

Segerstrom, S.C. (2000) Personality and the immune system: models, methods, and mechanisms. Ann. Behav. Med., 22, 180-190.

Siebel, W.A. (1994) Human Interaction. Glaser, Langwedel.

Siebel, W.A. \& Winkler, T. (1996) Noosomatik, Vol. V, 2nd Edn. Glaser, Langwedel.

Siebel, W.A. \& Winkler, T. (in press) Noosomatik, Vol. VI. Dareschta, Wiesbaden [available on CD-ROM].
Singh, R.B., Kartik, C., Otsuka, K., Pella, D. \& Pella, J. (2002) Brain-heart connection and the risk of heart attack. Biomed. Pharmacother, 56 (Suppl 2), $257-265$.

Smith, T.W., Glazer, K., Ruiz, J.M. \& Gallo, L.C. (2004) Hostility, anger, aggressiveness, and coronory heart disease: an interpersonal perspective on personality, emotion, and health. J. Personality, 72, 1217-1270.

Soria-Olivas, E., Martinez-Sober, M., Calpe-Maravilla, J., Guerrero-Martinez, J.F., Chorro-Gascó, J., \& Espi-López, J. (1998) Application of adaptive signal processing for determining the limits of $\mathrm{P}$ and $\mathrm{T}$ waves in an ECG. IEEE Trans. Biomed. Engng, 45, 1077-1080.

Stanton, A.L., Revenson, T.A. \& Tennen, H. (2007) Health psychology: psychological adjustment to chronic disease. Ann. Rev. Psych., 58, 565-592.

Stein, P.K., Bosner, M.S., Kleiger, R.E. \& Conger, B.M. (1994) Heart rate variability: a measure of cardiac autonomic tone. Am. Heart J., 127, 13761381.

Talairach, J. \& Tournoux, P. (1988) Co-Planar Stereotaxic Atlas of the Human Brain. Thieme, Stuttgart, Germany.

Taylor, G.J. (1984) Alexithymia: concept, measurement, and implications for treatment. Am. J. Psychiatry, 141, 725-732.

Taylor, G.J. \& Bagby, R.M. (2004) New trends in alexithymia research. Psychother. Psychosom., 73, 68-77.

Taylor, G.J., Bagby, R.M. \& Parker, J.D.A. (1992) The Revised Toronto Alexithymia Scale: some reliability, validity, and normative data. Psychother. Psychosom., 57, 34-41.

Verrier, R.L. \& Mittelman, M.A. (2000) The impact of emotions on the heart Prog. Brain Res., 122, 369-380.

Yeragani, V.K., Pohl, R., Berger, R., Balon, R., Ramesh, C., Glitz, D., Srinivasan, K. \& Weinberg, P. (1993) Decreased heart rate variability in panic disorder patients: a study of power-spectral analysis of heart rate. Psychiatry Res., 46, 89-103.

Yeragani, V.K., Pohl, R., Jampala, V.C., Balon, R., Ramesh, C. \& Srinivasan, K (2000) Increased QT variability in patients with panic disorder and depression. Psychiatry Res., 93, 225-235.

Yeragani, V.K., Rao, K.A., Smitha, M.R., Pohl, R.B., Balon, R. \& Srinivasan, K. (2002) Diminished chaos of heart rate time series in patients with major depression. Biol. Psychiatry, 51, 733-744.

Zuckerman, M., Buchsbaum, M.S. \& Murphy, D.L. (1980) Sensation seeking and its biological correlates. Psychol. Bull., 88, 187-214. 